\title{
Topological solitons of the Lawrence-Doniach model as equilibrium Josephson vortices in layered superconductors
}

\author{
Sergey V. Kuplevakhsky \\ B. Verkin Institute for Low Temperature Physics and Engineering of the National Academy of Science \\ of Ukraine, 47 Lenin Ave., Kharkov 61103, Ukraine \\ E-mail: kuplevakhsky@ilt.kharkov.ua
}

Received January 22, 2004

\begin{abstract}
We present a complete, exact solution of the problem of the magnetic properties of layered superconductors with an infinite number of superconducting layers in parallel fields $H>0$. Based on a new exact variational method, we determine the type of all stationary points of both the Gibbs and Helmholtz free-energy functionals. For the Gibbs free-energy functional, they are either points of strict, strong minima or saddle points. All stationary points of the Helmholtz free-energy functional are those of strict, strong minima. The only minimizes of both the functionals are the Meissner (0-soliton) solution and soliton solutions. The latter represent equilibrium Josephson vortices. In contrast, non-soliton configurations (interpreted in some previous publications as «isolated fluxons» and «fluxon lattices») are shown to be saddle points of the Gibbs free-energy functional: They violate the conservation law for the flux and the stationarity condition for the Helmholtz free-energy functional. For stable solutions, we give a topological classification and establish a one-to-one correspondence with Abrikosov vortices in type-II superconductors. In the limit of weak interlayer coupling, exact, closed-form expressions for all stable solutions are derived: They are nothing but the «vacuum state» and topological solitons of the coupled static sine-Gordon equations for the phase differences. The stable solutions cover the whole field range $0 \leq H<\infty$ and their stability regions overlap. Soliton solutions exist for arbitrary small transverse dimensions of the system, provided the field $H$ to be sufficiently high. Aside from their importance for weak superconductivity, the new soliton solutions can find applications in different fields of nonlinear physics and applied mathematics.
\end{abstract}

PACS: 74.50.+r, 74.80.Dm, 05.45.Yv

\section{Introduction}

In Ref. 1, concerned with a microscopic model, and Ref. 2, concerned with the phenomenological Lawrence-Doniach (LD) model [3], we have shown that the problem of the minimization of the Gibbs free-energy functional of layered superconductors with an infinite number of superconducting $(\mathrm{S})$ layers $(N=\infty)$ in parallel magnetic fields $H>0$ admits an exact solution. Advanced mathematical methods, employed in Refs. 1, 2, allowed us to overcome the complications related to mutual dependence of the phases of the $\mathrm{S}$ layers $\varphi_{n}$. (Unfortunately, these complications were not noticed in previous publications on the LD model
[4-9], which led to a loss of minimizers.) The main results of Refs. 1, 2 are worth recalling:

The set of minimizers derived in Refs. 1, 2 comprises the topologically trivial Meissner configuration and true soliton (vortex) configurations. As in the case of the Meissner state and Abrikosov vortices (i.e., the topological solitons [10-13] of the Ginzburg - Landau equations) in continuum type-II superconductors, all these configurations are characterized by the conserved topological index $N_{v}=0,1,2, \ldots$ (the vortex number, with $N_{v}=0$ for the Meissner state) and the conserved magnetic flux. For this reason, the topologically nontrivial solution with $N_{v}=1$ has been identified as an elementary Josephson vortex in layered superconductors at $H>0$. Physically, such a solution 
can be regarded as a bound state of interlayer flux quanta (one flux quantum per insulating layer). We have termed this solution «a vortex plane» because its field distribution has symmetry typical of plane defects.

The present paper complements and completes the investigation of Refs. 1, 2 in two major respects. First, we determine the type of all stationary points of the Gibbs free-energy functional, which allows us to classify all of the solutions available in the literature with regard to their stability. In particular, we show that, except for the Meissner solution, all non-soliton solutions (such as, e.g., «vortex lattices» $[6,7,14]$ ) correspond to saddle points. Second, in the limit of weak interlayer coupling, we derive exact, closed-form analytical expressions for the full set of stable solutions with $N_{v}=0,1,2, \ldots$. These solutions cover the whole field range $0 \leq H<\infty$, as they should, and include the results of Refs. 1, 2 as particular cases. They illustrate all the features of the Meissner state and vortex structure in weakly coupled superconductors (such as, e.g., an overlap of the stability regions and soliton nature of Josephson vortices) and establish true isomorphism with Abrikosov vortices in type-II superconductors. Moreover, they refute the erroneous belief $[15,16]$ that Josephson vortices «do not exist» in small (along the $\mathrm{S}$ layers) structures.

Although the issue of stability is crucial for the determination of the equilibrium vortex structure in layered superconductors, it has not been addressed in any previous publications*. Mathematically, a classification of stable solutions amounts to the determination of all points of local minima of the energy functionals. A local minimum of the Gibbs free-energy functional of layered superconductors is determined by the relations $[18,19]$

$$
\begin{gathered}
\delta \Omega\left[\delta f_{n}, \frac{d \delta f_{n}}{d y}, \delta \varphi_{n}, \frac{d \delta \varphi_{n}}{d y}, \delta \mathbf{A} ; H\right]_{\left\{\bar{f}_{n}, \bar{\varphi}_{n}, \overline{\mathbf{A}}\right\}}=0,(1) \\
\Omega\left[\bar{f}_{n}+\delta f_{n}, \frac{d \bar{f}_{n}}{d y}+\frac{d \delta f_{n}}{d y}, \bar{\varphi}_{n}+\delta \varphi_{n}, \frac{d \bar{\varphi}_{n}}{d y}+\frac{d \delta \varphi_{n}}{d y},\right. \\
\overline{\mathbf{A}}+\delta \mathbf{A} ; H] \geq \Omega\left[\bar{f}_{n}, \frac{d \bar{f}_{n}}{d y}, \bar{\varphi}_{n}, \frac{d \bar{\varphi}_{n}}{d y}, \overline{\mathbf{A}} ; H\right],
\end{gathered}
$$

where $\delta \Omega$ is the first variation of the Gibbs free-energy functional, induced by small variations $\delta f_{n}, \delta \varphi_{n}$, $\delta \mathbf{A}$ of the modulus of the order parameter $\left(\bar{f}_{n}\right)$, the phase $\left(\bar{\varphi}_{n}\right)$ and the vector potential $(\overline{\mathbf{A}})$, respectively $[1,2]$. [For example, numerical non-soliton solutions of Ref. 16 satisfy the stationarity condition (1) but do not meet the condition of the minimum (2).] The value of $\Omega$ on the right-hand side of (2) is associated with thermodynamic (observable) Gibbs free energy. The true equilibrium state corresponds to the minimum of the thermodynamic Gibbs free energy (i.e., the absolute minimum of the Gibbs free-energy functional) at a given $H>0$. The rest of the states, satisfying (1), (2) at a given $H>0$, are thermodynamically metastable: As an illustration, we refer to the Meissner state of the semi-infinite (along the layers) layered superconductor near the superheating field $H_{s}[1,2]$.

To eliminate any questions about the actual equilibrium field configurations in layered superconductors at $H>0$, we present an explicit mathematical proof that the Meissner (0-soliton) and vortex-plane (soliton) configurations are unique solutions that satisfy the conditions of the minimum (1), (2). Moreover, we show that all the minima are strict and strong [19]. For the sake of diversification, we employ a new method of exact minimization of the Gibbs free-energy functional that, in contrast to Refs. 1, 2, does not involve variation with respect to the phases $\varphi_{n}$ : The new method starts directly from the definition (1), (2) and automatically yields the conservation law for the flux and a full set of soliton boundary conditions. For definitiveness, we restrict ourselves to consideration of the popular LD model: Owing to the relationship [2] between the LD model and the true microscopic model of Ref. 1, all the results hold for the latter model as well. The paper is mostly concerned with mathematical aspects of the problem; all major derivations are given in full detail. As regards a comparison with the experiment, the reader is referred to Refs. 1, 2.

Section 2 of the paper is devoted to exact minimization of the Gibbs free-energy functional. In Sec. 2.1 we specify the geometry of the problem, introduce the Gibbs free-energy functional of the LD model, $\Omega_{L D}$, and discuss some of its properties. Using the conditions of the minimum, in Sec. 2.2 we reduce the problem of the minimization of $\Omega_{L D}$ to that of a simpler functional, $\Omega$, that possesses the same set of minimizers as $\Omega_{L D}$. In Sec. 2.3 we prove that the stationary points of $\Omega$ belong to two types: those of the minimum and saddle points. From the conditions of the minimum of $\Omega$ we derive the conservation law for

* Contrary to what is claimed, e.g., in Ref. 17, this issue cannot be resolved by comparing «characteristic length scales» or values of the energy functionals for distinct field configurations. The main problem is to establish whether the compared configurations locally minimize the energy functionals. 
the flux, which yields the Meissner and soliton (vortex-plane) solutions. The uniqueness of these solutions as minimizers of both $\Omega$ and $\Omega_{L D}$ is verified by the consideration of the exact lower bounds of the related Helmholz free-energy functional, $\Omega_{H}$, that explicitly depend on the conserved topological index $N_{v}$. In Sec. 2.4 we present a topological classification of the stable solutions and establish isomorphism between vortex planes in layered superconductors at $H>0$ and Abrikosov vortices in type-II superconductors.

In Sec. 3, in the limit of weak interlayer coupling, we derive exact, closed-form expressions for all stable solutions of the LD model (the Meissner and the soliton, or vortex, ones). The properties of these solutions are thoroughly investigated, all major limiting cases are considered. Isomorphism between vortex planes and ordinary Josephson vortices in the single junction is established.

The results obtained are summarized and discussed in Sec. 4. In particular, we explain where unstable non-soliton solutions come from. We also draw a comparison between our approach to layered superconductors and those of other authors. Mathematical flaws of these latter approaches are explicitly pointed out.

In Appendix A we discuss analytical properties and the solution of coupled static sine-Gordon (SG) equations for the phase differences, considered in some previous publications. We prove that the Meissner and vortex-plane solutions, derived in Sec. 3, are the unique stable solutions to the SG equations at $H>0$. We also establish a relationship to the exact variational principle of Refs. 1, 2. In Appendix B we verify the fulfillment of the Jacobi-Weierstrass-Hilbert sufficient condition for a strong minimum for the exact, closed-form solutions of Sec. 3. In Appendix C we draw a comparison between the soliton solutions of Sec. 3 and the non-soliton («lattice») solutions of Refs. 6, 7, 14, which serves as a good illustration of the general results of the main text.

\section{Exact minimization of the LD functional}

\subsection{Formulation of the problem}

The geometry of the problem is that of Refs. 1, 2: The layering axis is $x$, with $p$ being the period; the $y$ axis is directed along the $\mathrm{S}$ layers, with $-L \leq y \leq+L$ being the region occupied by the system [or $-\infty<y<+\infty$, if $L=\infty$ ]. A static, uniform external field is applied along the $z$ axis: $\mathbf{H}=(0,0, H \geq 0)$. The case of external current is not considered, i.e., $I=0$.

Under the assumption of homogeneity along the $z$ axis, we can write the Gibbs free-energy functional of the LD model as

$$
\begin{gathered}
\Omega_{L D}\left[f_{n}, \frac{d f_{n}}{d y}, \varphi_{n}, \frac{d \varphi_{n}}{d y}, \mathbf{A} ; H\right]=\frac{p H_{c}^{2}(T)}{4 \pi} W_{z} \times \\
\times \int_{-L}^{L} d y \sum_{n}\left\{-f_{n}^{2}(y)+12 f_{n}^{4}(y)+\zeta^{2}(T)\left[\frac{d f_{n}(y)}{d y}\right]^{2}+\right. \\
+\zeta^{2}(T)\left[\frac{d \varphi_{n}(y)}{d y}-2 e A_{y}(n p, y)\right]^{2} f_{n}^{2}(y)+ \\
+\frac{r(T)}{2}\left[f_{n-1}^{2}(y)+f_{n}^{2}(y)-2 f_{n}(y) f_{n-1}(y) \cos \Phi_{n, n-1}(y)\right]+ \\
\left.+\frac{4 e^{2} \zeta^{2}(T) \lambda^{2}(T)}{p} \int_{(n-1) p}^{n p} d x\left[\frac{\partial A_{y}(x, y)}{\partial x}-\frac{\partial A_{x}(x, y)}{\partial y}-H\right]^{2}\right\} \\
\Phi_{n, n-1}(y)=\varphi_{n}(y)-\varphi_{n-1}(y)-2 e \int_{(n-1) p}^{n p} d x A_{x}(x, y)= \\
=\phi_{n}(y)-2 e \int_{(n-1) p}^{n p} d x A_{x}(x, y) .
\end{gathered}
$$

Here $\hbar=c=1 ; \mathbf{A}=\left(A_{x}, A_{y}, 0\right)$ is the vector potential; $W_{z}$ is the length of the system in the $z$ direction $\left(W_{z} \rightarrow \infty\right) ; f_{n}(y)\left[0 \leq f_{n}(y) \leq 1\right]$ and $\varphi_{n}(y)$ are, respectively, the reduced modulus and the phase of the pair potential $\Delta_{n}(y)$ in the $n$th superconducting layer: $\Delta_{n}(y)=\Delta(T) f_{n}(y) \exp \phi_{n}(y) ; \phi_{n} \equiv \varphi_{n}-\varphi_{n-1}$; $H_{c}(T)$ is the thermodynamic critical field; $r(T)$ is a dimensionless phenomenological parameter of the Josephson interlayer coupling; $\zeta(T)$ and $\lambda(T)$ are the Ginzburg - Landau coherence length and the penetration depth, respectively. The local magnetic field $\mathbf{h}=(0,0, h)$ obeys the Maxwell equation

$$
h(x, y)=\frac{\partial A_{y}(x, y)}{\partial x}-\frac{\partial A_{x}(x, y)}{\partial y} .
$$

The variables $f_{n}, \varphi_{n}$, and $\mathbf{A}$ are subject to standard requirements [19]: $f_{n}, \varphi_{n}$ are supposed to be smooth in the whole interval $-L \leq y \leq L$, whereas $\mathbf{A}$ is piecewise smooth on the domain of definition, because at $x=n p$ only the continuity of $\mathbf{A}$ can be required. The summation in Eq. (3) runs over all the $\mathrm{S}$ layer indices $n$. To avoid mathematical complications related to the appearance of infinite sums while retaining the property of the periodicity of the barrier potential and the absence of outer boundaries in the $x$ direction, it is reasonable to compactify the model [20] by imposing periodic boundary conditions on observable quantities, 


$$
\begin{gathered}
f_{n+N}=f_{n}, \quad \Phi_{n+N, n+N-1}(y)=\Phi_{n, n-1}(y), \\
h(x+N p, y)=h(x, y), \\
\frac{d \varphi_{n+N}(y)}{d y}-2 e A_{y}[(n+N) p, y]= \\
=\frac{d \varphi_{n}(y)}{d y}-2 e A_{y}(n p, y),
\end{gathered}
$$

and proceed to the thermodynamic limit $\lim \Omega_{L D} / N=\omega_{L D}<\infty$ in the final expressions. (The existence of this limit will be proved in Sec. 2.3.) Using (6), we can write the total flux through the system as

$$
\begin{gathered}
\Phi=\int_{-L}^{L} d y \sum_{n} \int_{(n-1) p}^{n p} d x h(x, y)=\int_{-L}^{L} d y \sum_{i=1}^{N} \int_{(n-1+i) p}^{(n+i) p} d x h(x, y)= \\
=\int_{-L}^{L} d y\left[A_{y}[(n+N) p, y]-A_{y}(n p, y)\right]+ \\
+\sum_{i=1}^{N} \int_{(n-1+i) p}^{(n+i) p} d x\left[A_{x}(x,-L)-A_{x}(x, L)\right]= \\
=\frac{1}{2 e} \sum_{i=1}^{N}\left[\Phi_{n+i, n-1+i}(L)-\Phi_{n+i, n-1+i}(-L)\right] . \quad
\end{gathered}
$$

What we are going to do now is to find all sets of allowed field configurations $\left\{\bar{f}_{n}, \bar{\varphi}_{n}, \overline{\mathbf{A}}\right\}$ that at a given $H$ satisfy the condition of the minimum (2), i.e.,

$$
\begin{aligned}
& \Omega_{L D}\left[f_{n}, \frac{d f_{n}}{d y}, \varphi_{n}, \frac{d \varphi_{n}}{d y}, \mathbf{A} ; H\right] \geq \\
\geq & \Omega_{L D}\left[\bar{f}_{n}, \frac{d \bar{f}_{n}}{d y}, \bar{\varphi}_{n}, \frac{d \bar{\varphi}_{n}}{d y}, \overline{\mathbf{A}} ; H\right] \equiv \\
\equiv & \min \Omega_{L D}\left[f_{n}, \frac{d f_{n}}{d y}, \varphi_{n}, \frac{d \varphi_{n}}{d y}, \mathbf{A} ; H\right],
\end{aligned}
$$

where $\left\{f_{n}, \varphi_{n}, \mathbf{A}\right\}$ belong to sufficiently small neighborhood of $\left\{\bar{f}_{n}, \bar{\varphi}_{n}, \overline{\mathbf{A}}\right\}$.

\subsection{A new minimization method}

Our approach is standard [19] and consists in the determination of an exact lower bound of (3) at a given $H$ and finding the field configuration $\left\{\bar{f}_{n}, \bar{\varphi}_{n}, \overline{\mathbf{A}}\right\}$ that makes (3) equal to this lower bound. We begin with the stationarity condition (1) for $\Omega_{L D}$.
Variation with respect to $f_{n}$ immediately yields a set of equations

$$
\begin{gathered}
f_{n}(y)-f_{n}^{3}(y)+\zeta^{2}(T) \frac{d^{2} f_{n}(y)}{d y^{2}}= \\
=\frac{r(T)}{2}\left[2 f_{n}(y)-f_{n+1}(y) \cos \Phi_{n+1, n}(y)-\right. \\
\left.-f_{n-1}(y) \cos \Phi_{n, n-1}(y)\right]+ \\
+\zeta^{2}(T)\left[\frac{d \phi_{n}(y)}{d y}-2 e A_{y}(n p, y)\right]^{2} f_{n}(y)
\end{gathered}
$$

and boundary conditions

$$
\frac{d f_{n}}{d y}( \pm L)=0
$$

Variation with respect to $A_{x}$ leads to the Maxwell equation

$\frac{\partial h(x, y)}{\partial y}=4 \pi j_{n, n-1}(y) \equiv 4 \pi j_{0} f_{n}(y) f_{n-1}(y) \sin \Phi_{n, n-1}(y)$

in the regions $(n-1) p<x<n p$, and the boundary conditions

$$
h(x, \pm L)=H \text {. }
$$

In Eq. (11), the quantity $j_{n, n-1}(y)$ is the density of the Josephson current between the $(n-1)$ th and the $n$th layers, and $j_{0}=r(T) p / 16 \pi e \zeta^{2}(T) \lambda^{2}(T)$.

By variation with respect to $A_{y}$, we obtain the Maxwell equation

$$
\frac{\partial h(x, y)}{\partial x}=0
$$

in the regions $(n-1) p<x<n p$, and the boundary conditions at the $\mathrm{S}$ layers

$$
\begin{gathered}
h(n p-0, y)-h(n p+0, y)= \\
=\frac{p f_{n}^{2}(y)}{2 e \lambda^{2}(T)}\left[\frac{d \varphi_{n}(y)}{d y}-2 e A_{y}(n p, y)\right] .
\end{gathered}
$$

At this point, it is convenient to partially fix the gauge by the condition*

$$
A_{x}(x, y)=0, \quad A_{y}(x, y) \equiv A(x, y)
$$

which turns Eqs. (5), (11) and (13) into a system of linear inhomogeneous differential equations for $A(x, y)$ :

* This can be done by the gauge transformation $\mathbf{A}^{\prime}(x, y)=\mathbf{A}(x, y)+\nabla \psi(x, y), \quad \varphi_{n}^{\prime}(y)=\varphi_{n}(y)+2 e \psi(n p, y)$, where $\psi(x, y)=-\int_{C}^{x} d t A_{x}(t, y)$. 


$$
\begin{gathered}
\frac{\partial^{2} A(x, y)}{\partial y \partial x}=4 \pi j_{0} f_{n}(y) f_{n-1}(y) \sin \phi_{n}(y) \\
\frac{\partial^{2} A(x, y)}{\partial x^{2}}=0
\end{gathered}
$$

with the boundary conditions

$$
\begin{gathered}
A(n p-0, y)=A(n p+0, y), \\
\frac{\partial A}{\partial x}(n p-0, y)-\frac{\partial A}{\partial x}(n p+0, y)= \\
=\frac{p f_{n}^{2}(y)}{2 e \lambda^{2}(T)}\left[\frac{d \varphi_{n}(y)}{d y}-2 e A(n p, y)\right], \\
\frac{\partial A}{\partial x}(x, \pm L)=H .
\end{gathered}
$$

From (19), (20), we get the conditions of the vanishing of the intralayer currents at the outer boundaries

$$
\frac{d \varphi_{n}}{d y}( \pm L)-2 e A(n p, \pm L)=0
$$

and the conservation law for the total intralayer current

$$
\sum_{n} f_{n}^{2}(y)\left[\frac{d \varphi_{n}(y)}{d y}-2 e A(n p, y)\right]=0 .
$$

In Refs. 1, 2, relation (22) was employed for the minimization of (3) with respect to the phases $\varphi_{n}$.

The solution of (16), (17) under the conditions (18)-(20) is straightforward and has the form [2]

$$
\begin{gathered}
A(x, y)=\left[4 \pi j_{0} \int_{-L}^{y} d u f_{n}(u) f_{n-1}(u) \sin \phi_{n}(u)+H\right](x-n p)+\frac{1}{2 e} \frac{d \varphi_{n}(y)}{d y}- \\
-\frac{r(T)}{4 e \zeta^{2}(T)} \frac{1}{f_{n}^{2}(y)} \int_{-L}^{y} d u f_{n}(u)\left[f_{n-1}(u) \sin \phi_{n}(u)-f_{n+1}(u) \sin \phi_{n+1}(u)\right], \\
(n-1) p<x \leq n p,
\end{gathered}
$$

where

$$
\begin{gathered}
f_{n}(-y)=f_{n}(y), \\
\phi_{n}(-y)=-\phi_{n}(y)+2 \pi Z_{n}, \quad Z_{n}=0, \pm 1, \pm 2, \ldots,
\end{gathered}
$$

and the phase differences $\phi_{n} \equiv \varphi_{n}-\varphi_{n-1}$ obey the solvability conditions

$$
\begin{gathered}
\frac{d \phi_{n+1}(y)}{d y}=\frac{1}{\lambda_{J}^{2}} \int_{-L}^{y} d u f_{n+1}(u) f_{n}(u) \sin \phi_{n+1}(u)+2 e p H+ \\
+\frac{\varepsilon^{2}}{\lambda_{J}^{2}}\left[\frac { 1 } { f _ { n + 1 } ^ { 2 } ( y ) } \int _ { - L } ^ { y } d u f _ { n + 1 } ( u ) \left[f_{n}(u) \sin \phi_{n+1}(u)-\right.\right. \\
\left.-f_{n+2}(u) \sin \phi_{n+2}(u)\right]- \\
-\frac{1}{f_{n}^{2}(y)} \int_{-L}^{y} d u f_{n}(u)\left[f_{n-1}(u) \sin \phi_{n}(u)-\right. \\
\left.\left.-f_{n+1}(u) \sin \phi_{n+1}(u)\right]\right] .
\end{gathered}
$$

In Eqs. (26), we have introduced the Josephson penetration depth $\lambda_{J}=\left(8 \pi e j_{0} p\right)^{-1 / 2}$ and a dimen- sionless parameter $\varepsilon=p / \lambda$ [2]. By virtue of (24), (25), equations (26) yield

$$
\begin{gathered}
\frac{d \phi_{n+1}}{d y}( \pm L)=\frac{d \phi_{n}}{d y}( \pm L) \equiv \frac{d \phi}{d y}( \pm L), \\
\frac{d \phi}{d y}( \pm L)=2 e p H .
\end{gathered}
$$

For given $f_{n}$ obeying relations (24), equations (26) constitute a system of nonlinear integrodifferential equations for $\phi_{n}$, of first order with respect to differentiation. The formulation of the boundary value problem for these equations requires imposition of boundary conditions on $\phi_{n}$. Appropriate boundary conditions at $y=0$ are provided by the symmetry relations (25), i.e., the boundary conditions are

$$
\phi_{n}(0)=\pi Z_{n}, \quad Z_{n}=0, \pm 1, \pm 2, \ldots
$$

The main issue now is to find all solutions to (26), (29) that actually make (3) a minimum. As was shown in Refs. 1, 2, this issue is equivalent to exact minimization of (3) with respect to the phases $\varphi_{n}$. (See also Appendix A.) Below, we present an alternative, simpler method of exact minimization. 
Instead of minimizing with respect to $\varphi_{n}$, we introduce a new functional $\Omega$ via the relation

$$
\begin{gathered}
\Omega_{L D}\left[f_{n}, \frac{d f_{n}}{d y}, \varphi_{n}, \frac{d \varphi_{n}}{d y}, \mathbf{A} ; H\right]=\Omega\left[f_{n}, \frac{d f_{n}}{d y}, \varphi_{n}, \mathbf{A} ; H\right]+ \\
+\Omega_{0}+\frac{p H_{c}^{2}(T) \zeta^{2}(T) W_{z}}{4 \pi} \times \\
\quad \times \int_{-L}^{L} d y \sum_{n}\left[\frac{d \varphi_{n}(y)}{d y}-2 e A_{y}(n p, y)\right]^{2} f_{n}^{2}(y),(30)
\end{gathered}
$$

where

$$
\Omega_{0} \equiv \Omega_{L D}(0)=-\frac{H_{c}^{2}(T) p N L W_{z}}{4 \pi}
$$

is the $\mathrm{LD}$ free energy for $H=0$. The functional $\Omega$ will be considered on the same class of functions $f_{n}, \varphi_{n}, \mathbf{A}$ as the functional $\Omega_{L D}$ : In particular, these functions are supposed to satisfy conditions (14) at the internal boundaries and natural conditions at the outer boundaries $y= \pm L$.

The advantage of the new functional

$$
\begin{aligned}
& \Omega\left[f_{n}, \frac{d f_{n}}{d y}, \varphi_{n}, \mathbf{A} ; H\right]=\frac{p H_{c}^{2}(T)}{4 \pi} W_{z} \int_{-L}^{L} d y \sum_{n}\left[\frac{1}{2}\left[1-f_{n}^{2}(y)\right]^{2}+\zeta^{2}(T)\left[\frac{d f_{n}(y)}{d y}\right]^{2}+\frac{r(T)}{2}\left[f_{n-1}^{2}(y)+\right.\right. \\
& \left.\left.+f_{n}^{2}(y)-2 f_{n}(y) f_{n-1}(y) \cos \Phi_{n, n-1}(y)\right]+\frac{4 e^{2} \zeta^{2}(T) \lambda^{2}(T)}{p} \int_{(n-1) p}^{n p} d x\left[\frac{\partial A_{y}(x, y)}{\partial x}-\frac{\partial A_{x}(x, y)}{\partial y}-H\right]^{2}\right]
\end{aligned}
$$

is that it has simple properties: The minimization of (32) does not require variation with respect to $\varphi_{n}$. Moreover, a local minimum of $\Omega$ at a given $H, \min \Omega$, provides a lower bound for $\Omega_{L D}$.

Indeed, the functional $\Omega$ is positive, i.e.,

$$
\Omega\left[f_{n}, \frac{d f_{n}}{d y}, \varphi_{n}, \mathbf{A} ; H\right] \geq 0,
$$

and its exact lower bound at $H=0$, $\inf \Omega=0$, is achieved on the field configurations

$$
\bar{f}_{n}(y) \equiv 1, \quad \bar{\varphi}_{n}(y) \equiv \psi(y), \quad \bar{A}_{x} \equiv 0, \quad \bar{A}_{y}=\frac{d \psi}{d y},
$$

where $\psi(y)$ is an arbitrary smooth function. [Note that the exact lower bound of (3) at $H=0$, inf $\Omega_{L D}=\Omega_{0}$, is achieved on the same field configurations (34).] Because of (33), the continuous functional (32) necessarily has a minimum at any $H>0$ in the allowed class of functions, specified above [19]. By virtue of (30) and the definition of the minimum,

$$
\begin{aligned}
& \Omega_{L D}\left[f_{n}, \frac{d f_{n}}{d y}, \varphi_{n}, \frac{d \varphi_{n}}{d y}, \mathbf{A} ; H\right] \geq \\
& \geq \Omega\left[f_{n}, \frac{d f_{n}}{d y}, \varphi_{n}, \mathbf{A} ; H\right]+\Omega_{0} \geq \\
& \geq \min \Omega\left[f_{n}, \frac{d f_{n}}{d y}, \varphi_{n}, \mathbf{A} ; H\right]+\Omega_{0},
\end{aligned}
$$

where the right-hand side of the second inequality provides the desired lower bound for $\Omega_{L D}$ at a given $H$.
To determine $\min \Omega$, we first find all field configurations $\left\{f_{n}, \varphi_{n}, \mathbf{A}\right\}$ that satisfy the stationarity condition (1). Variation with respect to $f_{n}$ yields the equations

$$
\begin{gathered}
f_{n}(y)-f_{n}^{3}(y)+\zeta^{2}(T) \frac{d^{2} f_{n}(y)}{d y^{2}}= \\
=\frac{r(T)}{2}\left[2 f_{n}(y)-f_{n+1}(y) \cos \Phi_{n+1, n}(y)-\right. \\
\left.-f_{n-1}(y) \cos \Phi_{n, n-1}(y)\right]
\end{gathered}
$$

and boundary conditions (10). Variation with respect to $A_{x}$ leads to the Maxwell equation (11) in the regions $(n-1) p<x<n p$, and the boundary conditions (12). By variation with respect to $A_{y}$, we obtain the Maxwell equation (13) in the regions $(n-1) p<x<n p$, and new boundary conditions at the $\mathrm{S}$ layers,

$$
h(n p-0, y)=h(n p+0, y) .
$$

Application of (37) to (14), in turn, yields

$$
\frac{d \varphi_{n}(y)}{d y}-2 e A(n p, y)=0
$$

Combining (14) with (13), we get

$$
h(x, y) \equiv h(y),
$$

which, upon substitution into (11), results in

$$
f_{n-1}(y) \sin \Phi_{n, n-1}(y)=f_{n+1}(y) \sin \Phi_{n+1, n}(y) .
$$


Relations (40) reflect the continuity of the Josephson currents at $x=n p$ and constitute a consequence of $U(1)$ gauge symmetry: They can be obtained directly, by varying (32) with respect to $\varphi_{n}[1,2]$. In view of (39), the solution of these relations is straightforward:

$$
f_{n}(y) \equiv f(y), \quad \Phi_{n, n-1}(y) \equiv \phi(y)-2 e \int_{0}^{p} d x A_{x}(x, y)
$$

Note that relations (39), (41) identically satisfy the periodic boundary conditions (6). According to (41), the phases $\varphi_{n}$ obey the finite difference equation $\varphi_{n+1}-2 \varphi_{n}+\varphi_{n-1}=0$ with the boundary condition $\varphi_{n}-\varphi_{n-1}=\phi$, whose solution is [21]

$$
\varphi_{n}(y)=n \phi(y)+\psi(y),
$$

where $\psi(y)$ is an arbitrary smooth function.

Our next course of action follows the steps leading to Eqs. (23)-(29): We fix the gauge by (15) and solve the resulting equations for $A(x, y)$. As a consequence, in addition to (42), we arrive at coupled equations for $f$ and $\phi$,

$$
\begin{gathered}
f(y)-f^{3}(y)+\zeta^{2}(T) \frac{d^{2} f(y)}{d y^{2}}+ \\
+r(T)[1-\cos \phi(y)] f(y)=0, \quad \frac{d f}{d y}( \pm L)=0, \\
\frac{d \phi(y)}{d y}=\frac{1}{\lambda_{J}^{2}} \int_{-L}^{y} d u f^{2}(u) \sin \phi(u)+2 e p H, \\
\phi(-y)=-\phi(y)+2 \pi Z, \quad Z=0, \pm 1, \pm 2, \ldots
\end{gathered}
$$

and explicit expressions

$$
\begin{gathered}
A(x, y)=\frac{1}{2 e p} \frac{d \phi(y)}{d y} x+\frac{1}{2 e p} \frac{d \psi(y)}{d y}, \\
h(y)=\frac{1}{2 e p} \frac{d \phi(y)}{d y} .
\end{gathered}
$$

Any minimizer $\left\{\bar{f}_{n}, \bar{\varphi}_{n}, \overline{\mathbf{A}}\right\}$ of (32), at a given $H$, necessarily satisfies (15) and (41)-(47). At the same time, this minimizer automatically satisfies (9)-(14) and (16)-(28). Moreover, as can be easily verified by direct substitution,

$$
\begin{gathered}
\Omega_{L D}\left[\bar{f}, \frac{d \bar{f}}{d y}, \bar{\varphi}_{n}, \overline{\mathbf{A}} ; H\right]=\Omega\left[\bar{f}, \frac{d \bar{f}}{d y}, \bar{\varphi}_{n}, \overline{\mathbf{A}} ; H\right]+\Omega_{0}= \\
=\min \Omega\left[f_{n}, \frac{d f_{n}}{d y}, \varphi_{n}, \mathbf{A} ; H\right]+\Omega_{0} .
\end{gathered}
$$

According to (35) and the definition of the minimum (8), this means that

$$
\begin{gathered}
\Omega_{L D}\left[\bar{f}, \frac{d \bar{f}}{d y}, \bar{\varphi}_{n}, \overline{\mathbf{A}} ; H\right]= \\
=\inf \Omega_{L D}\left[f_{n}, \frac{d f_{n}}{d y}, \varphi_{n}, \frac{d \varphi_{n}}{d y}, \mathbf{A} ; H\right]= \\
=\min \Omega_{L D}\left[f_{n}, \frac{d f_{n}}{d y}, \varphi_{n}, \frac{d \varphi_{n}}{d y}, \mathbf{A} ; H\right]
\end{gathered}
$$

i.e., any set $\left\{\bar{f}_{n}, \bar{\varphi}_{n}, \overline{\mathbf{A}}\right\}$ that in the gauge (15) satisfies (41)-(47) and minimizes (32) is a minimizer of (3). On the other hand, for any set $\left\{f_{n}, \varphi_{n}, \mathbf{A}\right\}$ that in the gauge (15) does not satisfy (41)-(47) we have

$$
\begin{gathered}
\Omega_{L D}\left[f_{n}, \frac{d f_{n}}{d y}, \varphi_{n}, \frac{d \varphi_{n}}{d y}, \mathbf{A} ; H\right]> \\
>\Omega\left[f_{n}, \frac{d f_{n}}{d y}, \varphi_{n}, \mathbf{A} ; H\right]+\Omega_{0} \geq \\
\geq \min \Omega\left[f_{n}, \frac{d f_{n}}{d y}, \varphi_{n}, \mathbf{A} ; H\right]+\Omega_{0}= \\
=\Omega\left[\bar{f}, \frac{d \bar{f}}{d y}, \bar{\varphi}_{n}, \mathbf{A} ; H\right]+\Omega_{0}= \\
=\Omega_{L D}\left[\bar{f}, \frac{d \bar{f}}{d y}, \bar{\varphi}_{n}, \overline{\mathbf{A}} ; H\right]= \\
=\min \Omega_{L D}\left[f_{n}, \frac{d f_{n}}{d y}, \varphi_{n}, \frac{d \varphi_{n}}{d y}, \mathbf{A} ; H\right] .
\end{gathered}
$$

A strict inequality in the first line of (50) means that the minimizer $\left\{\bar{f}_{n}, \bar{\varphi}_{n}, \overline{\mathbf{A}}\right\}$ makes (3) a strict minimum [19]. [Note that the gauge (15) was employed here for the sake of convenience only: It allowed us to obtain an explicit solution for $\mathbf{A}$ by simple means.]

Summarizing, we have proved the following: A set $\left\{\bar{f}_{n}, \bar{\varphi}_{n}, \overline{\mathbf{A}}\right\}$ minimizes the LD functional (3) if and only if it is a minimizer of the functional (32) and, hence, necessarily satisfies the symmetry relations (41), with the resulting local magnetic field obeying (39). In the next subsection, we will show that a full set of the minimizers of (32), and, respectively, of (3), comprises the soliton (vortex-plane) solutions and the Meissner (0-soliton) solution [1,2].

\subsection{The conservation law for the flux and soliton boundary conditions}

To determine all the minimizers of (32), we first rewrite this functional as follows: 


$$
\begin{gathered}
\Omega\left[f, \frac{d f}{d y}, \phi, \frac{d \phi}{d y} ; H\right]= \\
=\frac{N p W W_{z} H^{2}}{8 \pi}+\Omega_{H}\left[f, \phi, \frac{d \phi}{d y}\right]-\frac{H \Phi W_{z}}{4 \pi},
\end{gathered}
$$

where

$$
\begin{gathered}
\Omega_{H}\left[f, \frac{d f}{d y}, \phi, \frac{d \phi}{d y}\right]= \\
=\frac{N p H_{c}^{2}(T)}{4 \pi} W_{z} \int_{-L}^{L} d y\left[\frac{1}{2}\left[1-f^{2}(y)\right]^{2}+\zeta^{2}(T)\left[\frac{d f(y)}{d y}\right]^{2}+\right. \\
\left.+r(T)\left[[1-\cos \phi(y)] f^{2}(y)+\frac{\lambda_{J}^{2}}{2}\left[\frac{d \phi(y)}{d y}\right]^{2}\right]\right], \text { (52) }
\end{gathered}
$$

and the total flux (7), in view of (15) and (41), is given by

$$
\Phi=\frac{N}{2 e}[\phi(L)-\phi(-L)] \equiv N \Phi_{0} \frac{1}{2 \pi} \int_{-L}^{L} d y \frac{d \phi(y)}{d y},
$$

with $\Phi_{0}=\pi / e$ being the flux quantum. Note that the first term on the right-hand side of (51) is merely the energy of the magnetic field in the absence of Josephson coupling.

Note that both $\Omega$ and $\Omega_{H}$ explicitly satisfy Legendre's necessary condition of the strong minimum [19]:

$$
\begin{gathered}
\frac{\delta^{2} \Omega(H)}{[\delta(d f / d y)]^{2}} \equiv \frac{N p H_{c}^{2}(T) W_{z}}{2 \pi} \zeta^{2}(T)>0, \\
\frac{\delta^{2} \Omega_{(H)}}{[\delta(d \phi / d y)]^{2}} \equiv \frac{N p H_{c}^{2}(T) W_{z}}{4 \pi} r(T) \lambda_{J}^{2}(T)>0, \\
\frac{\delta^{2} \Omega_{(H)}}{\delta(d f / d y) \delta(d \phi / d y)}=\frac{\delta^{2} \Omega_{(H)}}{\delta(d \phi / d y) \delta(d f / d y)} \equiv 0 .
\end{gathered}
$$

Therefore, all stationary points of $\Omega, \Omega_{H}$ are either strong minima or saddle points.

The stationarity condition for both $\Omega$ and $\Omega_{H}$ requires that first variations with respect to $f$ and $\phi$ vanish [compare with (1)]. Variation with respect to $f$ yields Eqs. (43), as expected. Consider now the first variation of $\Omega$ and $\Omega_{H}$ with respect to $\phi$ :

$$
\begin{gathered}
\delta \Omega\left[\delta f, \frac{d \delta f}{d y}, \delta \phi, \frac{d \delta \phi}{d y} ; H\right]= \\
=\delta \Omega_{H}\left[\delta f, \frac{d \delta f}{d y}, \delta \phi, \frac{d \delta \phi}{d y}\right]-\frac{H W_{z}}{4 \pi} \delta \Phi,
\end{gathered}
$$

$$
\begin{gathered}
\delta \Omega_{H}\left[\delta f, \frac{d \delta f}{d y}, \delta \phi, \frac{d \delta \phi}{d y}\right]=\frac{N p H_{c}^{2}(T) W_{z} r(T)}{4 \pi} \times \\
\times \int_{-L}^{L} d y\left[f^{2}(y) \sin \phi(y)-\lambda_{J}^{2} \frac{d^{2} \phi(y)}{d y^{2}}\right] \delta \phi(y)+ \\
+\frac{1}{e p} \frac{d \phi}{d y}(-L) \frac{W_{z}}{8 \pi} \delta \Phi
\end{gathered}
$$

where the variation of the flux is

$$
\delta \Phi=\frac{N}{2 e}[\delta \phi(L)-\delta \phi(-L)] \equiv N \Phi_{0} \delta\left(\frac{1}{2 \pi} \int_{-L}^{L} d y \frac{d \phi(y)}{d y}\right) .
$$

The requirement of the vanishing of the volume variation in both (55) and (56) yields

$$
\frac{d^{2} \phi(y)}{d y^{2}}=\frac{f^{2}(y)}{\lambda_{J}^{2}} \sin \phi(y),
$$

which is a mere consequence of (44). However, the requirement of stationarity with respect to surface variation [which is proportional to the variation of the flux (57)] is stronger for (56) than for (55). The surface variation in (55) cancels out owing to the boundary conditions (28). In contrast, conditions (28) do not ensure the vanishing of the surface variation in (56), and the stationarity of $\Omega_{H}$ at $H>0$ requires

$$
\delta \Phi=0
$$

or, equivalently,

$$
\Phi=N \Phi_{0} \frac{1}{2 \pi} \int_{-L}^{L} d y \frac{d \phi(y)}{d y}=\text { const } \geq 0,
$$

where the inequality sign corresponds to $H \geq 0$.

Note that higher variations of $\Omega$ and $\Omega_{H}$ are equal to each other: $\delta^{k} \Omega=\delta^{k} \Omega_{H}(k \geq 2)$. Thus, all the minimizers of $\Omega_{H}$ also minimize $\Omega$. On the other hand, the functional $\Omega$ has no minimizers other than those that simultaneously minimize $\Omega_{H}$. Indeed, let $\{\bar{f}, \bar{\phi}\}$ be a minimizer of $\Omega$ in a class of trial functions that admit arbitrary variations $\delta \Phi$. Then $\{\bar{f}, \bar{\phi}\}$ is necessarily a minimizer of $\Omega$ in a subclass of trial functions that satisfy (59). From the condition for the minimum of $\Omega$

$$
\begin{gathered}
\Omega\left[\bar{f}+\delta f, \frac{d \bar{f}}{d y}+\frac{d \delta f}{d y}, \bar{\phi}+\delta \phi, \frac{d \bar{\phi}}{d y}+\frac{d \delta \phi}{d y} ; H\right] \geq \\
\geq \Omega\left[\bar{f}, \frac{d \bar{f}}{d y}, \bar{\phi}, \frac{d \bar{\phi}}{d y} ; H\right]
\end{gathered}
$$


[compare with (2)] on this subclass of trial functions, we have

$$
\begin{gathered}
\Omega_{H}\left[\bar{f}+\delta f, \frac{d \bar{f}}{d y}+\frac{d \delta f}{d y}, \bar{\phi}+\delta \phi, \frac{d \bar{\phi}}{d y}+\frac{d \delta \phi}{d y}\right]- \\
-\Omega_{H}\left[\bar{f}, \frac{d \bar{f}}{d y}, \bar{\phi}, \frac{d \bar{\phi}}{d y}\right]-\frac{H W_{z}}{4 \pi} \delta \Phi= \\
=\Omega_{H}\left[\bar{f}+\delta f, \frac{d \bar{f}}{d y}+\frac{d \delta f}{d y}, \bar{\phi}+\delta \phi, \frac{d \bar{\phi}}{d y}+\frac{d \delta \phi}{d y}\right]- \\
-\Omega_{H}\left[\bar{f}, \frac{d \bar{f}}{d y}, \bar{\phi}, \frac{d \bar{\phi}}{d y}\right] \geq 0,
\end{gathered}
$$

which is the condition of the minimum of $\Omega_{H}$. For this reason, it is sufficient to find all the minimizers of $\Omega_{H}$.

Physically, conditions (59), (60) ensure the stability of the flux $\Phi$ against any small perturbations, represented by the variations $\bar{\phi} \rightarrow \bar{\phi}+\delta \phi$, which is a manifestation of the Meissner effect [22]. Conditions (59), (60) also imply that $\Phi$ plays the role of a thermodynamic variable in (51), which, in turn, allows us to identify $\Omega_{H}$ as the Helmholz free-energy functional.

Now we will derive the Meissner (0-soliton) and vortex-plane (soliton) boundary conditions [1,2] from the conservation law for the flux (59), (60). As a starting point, we note that all the extremals of $\Omega_{H}$ that satisfy (58), (28) possess the symmetry properties (45). Since trial functions of this type take on only discrete values at $y=0, \phi(0)=\pi Z$, the requirement of continuity of variations $\delta \phi$ imposes the constraint

$$
\delta \phi(0)=\pi \delta Z=0 .
$$

Equations (57), (59), in turn, yield

$$
\delta \phi(L)=\delta \phi(-L) \text {. }
$$

On the other hand, relations (45), applied at $y=L$, by virtue of (63) yield

$$
\delta \phi(L)=-\delta \phi(-L) .
$$

Combining (64) with (65), we arrive at the conditions

$$
\delta \phi( \pm L)=0 .
$$

Using (60), we write:

$$
\frac{\phi(L)-\phi(-L)}{2 \pi}=\frac{1}{2 \pi} \int_{-L}^{L} d y \frac{d \phi(y)}{d y}=
$$

$$
=\left[\frac{1}{2 \pi} \int_{-L}^{L} d y \frac{d \phi(y)}{d y}\right]+\left\{\frac{1}{2 \pi} \int_{-L}^{L} d y \frac{d \phi(y)}{d y}\right\}=\text { const } \geq 0,
$$

where $[x]$ and $\{x\}$ are, respectively, the integer and fractional parts of $x[0 \leq\{x\}<1]$. On the other hand, taking account of (45) and (63), (66), we have

$$
\frac{\phi(L)-\phi(-L)}{2 \pi}=Z-\frac{\phi(-L)}{\pi}=\text { const } \geq 0 .
$$

A comparison of (67) with (68) leads to the identification

$$
\begin{gathered}
\frac{\phi(0)}{\pi}=Z=N_{v} \equiv\left[\frac{1}{2 \pi} \int_{-L}^{L} d y \frac{d \phi(y)}{d y}\right] \geq 0, \\
-\frac{\phi(-L)}{\pi} \equiv\left\{\frac{1}{2 \pi} \int_{-L}^{L} d y \frac{d \phi(y)}{d y}\right\},-\pi<\phi(-L) \leq 0 .
\end{gathered}
$$

Relations (69), (70) are exactly the Meissner $\left(N_{v}=0\right)$ and vortex-plane $\left(N_{v}=1,2, \ldots\right)$ boundary conditions employed in Refs. 1, 2. The conserved topological index $N_{v}=0,1,2, \ldots$ has a clear meaning of the number of solitons (i.e., vortex planes or the Josephson vortices) at $H>0$. The conserved flux (60), rewritten via the conserved quantities $N_{v}$ and $\phi(-L)$, takes the form

$$
\Phi=N \Phi_{0}\left[N_{v}+\frac{|\phi(-L)|}{\pi}\right] .
$$

As follows from (71), the solution with given $N_{v}$ appears when

$$
\phi(-L)=0, \quad \phi(0)=\pi N_{v}, \quad \phi(-L)=2 \pi N_{v},
$$

which corresponds to the minimum of the density of the Josephson energy [the third term on the righthand side of (52)] at the boundaries $y= \pm L$. This solution vanishes when

$$
\phi( \pm L)= \pm \pi, \quad \phi_{n}(0)=\pi N_{v},
$$

which corresponds to the maximum of the density of the Josephson energy at $y= \pm L$ and saddle-point instability. By (28), conditions (72), (73) determine, respectively, the exact lower and upper bounds of the stability regions of the Meissner $\left(N_{v}=0\right)$ and vortex $\left(N_{v}=1,2, \ldots\right)$ configurations in the external field $H \geq 0$.

We will show now that conditions (69), (70) for the SG equations (58) together with (44) actually specify all the minimizers of (52) [and, correspondingly, of (51)]. Indeed, let $\bar{f}(y)$ be an arbitrary smooth function that for $y \in[-L, L]$ satisfies the condi- 
tions $0<\bar{f} \leq 1,(d \bar{f} / d y)( \pm L)=0$, and $\bar{f}_{0} \equiv \min \bar{f}(y)$. Using elementary inequalities $a+b \geq 2 \sqrt{a b}(a, b>0)$ and $-|q| \leq q \leq|q|$, we derive a sequence of inequalities for (52) with $f=\bar{f}$ :

$$
\begin{gathered}
\Omega_{H}\left[f, \frac{d \bar{f}}{d y}, \phi, \frac{d \phi}{d y}\right] \geq C+\frac{N p H_{c}^{2}(T) W_{z} r(T)}{4 \pi} \times \\
\times \int_{-L}^{L} d y\left[\bar{f}_{0}^{2}[1-\cos \phi(y)]+\frac{\lambda_{J}^{2}}{2}\left[\frac{d \phi(y)}{d y}\right]^{2}\right] \geq \\
\geq C+\frac{N p H_{c}^{2}(T) W_{z} r(T) \lambda_{J} \bar{f}_{0}}{4 \pi} \int_{-L}^{L} d y\left|\sin \frac{\phi(y)}{2}\right|\left|\frac{d \phi(y)}{d y}\right| \geq \\
\geq C+\frac{2 N p H_{c}^{2}(T) W_{z} r(T) \lambda_{J} \bar{f}_{0}}{\pi}\left[N_{v}+1-\cos \frac{\phi(-L)}{2}\right],
\end{gathered}
$$

where

$$
\begin{gathered}
C \equiv \frac{N p H_{c}^{2}(T) W_{z}}{4 \pi} \times \\
\times \int_{-L}^{L} d y\left[\frac{1}{2}\left[1-\bar{f}^{2}(y)\right]^{2}+\zeta^{2}(T)\left[\frac{d \bar{f}(y)}{d y}\right]^{2}\right] \geq 0 .
\end{gathered}
$$

Inequalities of the type (74) are employed in soliton theory to prove the existence and stability of soliton solutions [10-13,23]. In our case, inequality (74) shows that (52) has an exact lower bound in the class of functions $\{f, \phi\}$ parameterized by the conserved quantities $N_{v}$ and $\phi(-L)$ :

$$
\begin{gathered}
\inf \Omega_{H}\left[f, \frac{d f}{d y}, \phi, \frac{d \phi}{d y}\right]=\mu\left[N_{v}, \phi(-L)\right], \\
\mu\left[N_{v}, \phi(-L)\right] \geq \\
\geq \frac{2 N p H_{c}^{2}(T) W_{z} r(T) \lambda_{J} f_{*}}{\pi}\left[N_{v}+1-\cos \frac{\phi(-L)}{2}\right],
\end{gathered}
$$

where $f_{*}>0$ is the same for all the sets $\left\{N_{v}, \phi(-L)\right\}$.

In view of the continuity of $\Omega_{H}$ [19], the exact lower bound (75) is achieved on the corresponding solution $\{\bar{f}, \bar{\phi}\}$ to (43), (58), (69), and (70). According to Sec. 2.2., this solution represents the desired minimizer of (3). Given that for any minimizer

$$
\Omega_{L D}\left[\bar{f}, \frac{d \bar{f}}{d y}, \bar{\varphi}_{n}, \overline{\mathbf{A}} ; H\right]=N \omega_{L D}\left[\bar{f}, \frac{d \bar{f}}{d y}, \bar{\varphi}_{n}, \overline{\mathbf{A}} ; H\right],
$$

where $\omega_{L D}<\infty$, we have thus proved the existence of the thermodynamic limit for $N \rightarrow \infty$.
For practical applications, we note the first integral of Eqs. (43), (58) that immediately follows from (52):

$$
\begin{gathered}
\zeta^{2}(T)\left[\frac{d f}{d y}\right]^{2}+\frac{r(T) \lambda_{J}^{2}}{2}\left[\frac{d \phi}{d y}\right]^{2}+ \\
+f^{2}-\frac{1}{2} f^{4}-r(T)[1-\cos \phi] f^{2}=C,
\end{gathered}
$$

where the constant of integration can be determined from the boundary conditions at $y=-L$ :

$$
\begin{aligned}
C= & 2 e^{2} p^{2} \lambda_{J}^{2} H^{2}+f^{2}(-L)-\frac{1}{2} f^{4}(-L)- \\
& -r(T)[1-\cos \phi(-L)] f^{2}(-L) .
\end{aligned}
$$

\subsection{A topological classification and isomorphism to Abrikosov vortices in type-II superconductors}

The above results can be given a very clear interpretation within the framework of the theory of topological defects in continuum media $[10-13,24]$. Consider the thermodynamic LD free energy, obtained by the substitution of a minimizer $\left\{\bar{f}, \bar{\varphi}_{n}, \overline{\mathbf{A}}\right\}$ into (3):

$$
\begin{gathered}
\Omega_{L D}(H)=\Omega_{0}+\frac{N p W W_{z} H^{2}}{8 \pi}-\frac{H \Phi W_{z}}{4 \pi}+ \\
+\frac{N p H_{c}^{2}(T) W_{z}}{4 \pi} \int_{-L}^{L} d y\left[\frac{1}{2}\left[1-\bar{f}^{2}(y)\right]^{2}+\zeta^{2}(T)\left[\frac{d \bar{f}(y)}{d y}\right]^{2}+\right. \\
\left.+r(T)\left[\bar{f}^{2}(y)[1-\cos \bar{\phi}(y)]+\frac{\lambda_{J}^{2}}{2}\left[\frac{d \bar{\phi}(y)}{d y}\right]^{2}\right]\right] . \quad \text { (78) }
\end{gathered}
$$

Owing to the symmetry relations (24), (41), (45) and the boundary conditions (10), (28), the density of $\Omega_{L D}(H)$ is equal at $y=-L$ and $y=+L$ and thus corresponds to the degenerate equilibrium ( $\ll$ vacuum») state, unperturbed by topological defects (solitons). Mathematically, the boundary of the interval $-L \leq y \leq L$ can be considered as a 0 -dimensional sphere: $S^{0}=\{-L,+L\}$. Given that configurations $\bar{\phi}$ and $\bar{\phi}+2 \pi Z(Z=0, \pm 1, \pm 2, \ldots)$ are physically indistinguishable, we can fix the values $\bar{\phi}(-L)$ as in (70) and regard the functions

$$
\psi(+L) \equiv \frac{\bar{\phi}(+L)+\bar{\phi}(-L)}{2 \pi}=Z_{n} \equiv\left[\frac{1}{2 \pi} \int_{-L}^{L} d y \frac{d \bar{\phi}(y)}{d y}\right]
$$

as continuous maps of the boundary into the additive group of the integers, $\mathbf{Z}: S^{0} \stackrel{\Psi}{\rightarrow} \mathbf{Z}$. ( $\mathbf{Z}$ is the group of the degeneracy of the equilibrium state, or the order-parameter space.) The fact of the existence of to- 
pologically nontrivial maps of this type, realized by soliton solutions, can be expressed in terms of the zeroth homotopy group [10-13,24] $\pi_{0}(M)$, where the index $\ll 0 »$ stands for the boundary $S^{0}$ and $M$ is the order-parameter space:

$$
\pi_{0}(\mathbf{Z})=\mathbf{Z} .
$$

The external field $H>0$ breaks the symmetry $\bar{\phi} \rightarrow-\bar{\phi}$ [see the third term on the right-hand side of (78)]. Therefore, only the values $Z \equiv N_{v}=0,1,2, \ldots$ are allowed, with $N_{v}=0$ being the «vacuum» Meissner state. In this way, we arrive at a natural classification of the minimizers of (3) with respect to the conserved topological (vortex) number $N_{v}$.

Note that in the case of Abrikosov vortices in continuum type-II superconductors the boundary is topologically equivalent to the circle $S^{1}$, the order-parameter space is also the circle: $M \equiv S^{1}[10-13]$. Thus, the pertinent homotopy group is the fundamental group of the circle:

$$
\pi_{1}\left(S^{1}\right)=\mathbf{Z} .
$$

Since in the presence of an external field $H>0$ the topological indices for Abrikosov vortices take on the values $Z \equiv N_{v}=0,1,2, \ldots$, relations (80) and (81) establish an isomorphism between the vortex structure in type-II superconductors and that in layered superconductors at $H>0$, with $N_{v}=0$ for the Meissner state and a single Abrikosov vortex $\left(N_{v}=1\right)$ standing in a one-to-one correspondence with a single vortex plane (not an «isolated fluxon» as is claimed in previous publications [41]).

\section{The exact, closed-form solution for $r(T)<<1$ and isomorphism to Josephson vortices in the single junction}

Equations (43) and (58) with the soliton boundary conditions (69) and (70) can be solved by perturbation methods for arbitrary values of the interlayer coupling $r(T)$. However, of particular interest is the limit of weak coupling, $r(T)<<1$.

In the case $r(T)<<1$, the zeroth-order solution to (43) has the form $f_{0} \equiv 1$. Upon substitution into (58), we obtain the well-known static sine-Gordon equation:

$$
\frac{d^{2} \phi(y)}{d y^{2}}=\frac{1}{\lambda_{J}^{2}} \sin \phi(y) .
$$

Under the conditions (69), (70), the exact solution to (82) is

$$
\phi(y)=\pi\left(N_{v}-1\right)+2 \mathrm{am}\left(\frac{y}{k \lambda_{J}}+K\left(k^{2}\right), k\right),(83
$$

$$
\begin{gathered}
\operatorname{dn}\left(\frac{L}{k \lambda_{J}}, k\right)=\frac{\sqrt{1-k^{2}}}{k} \frac{H_{s}}{H}, \quad N_{v}=2 m, m=0,1, \ldots ; \\
\phi(y)=\pi N_{v}+2 \operatorname{am}\left(\frac{y}{k \lambda_{J}}, k\right)
\end{gathered}
$$

$$
\operatorname{dn}\left(\frac{L}{k \lambda_{J}}, k\right)=k \frac{H}{H_{S}}, N_{v}=2 m+1, m=0,1, \ldots,
$$

where am $(u)$ and $\operatorname{dn}(u)=(d / d u)$ am $(u)$ are the Jacobian elliptic functions, and $K\left(k^{2}\right)$ is the elliptic integral of the first kind [25].

The stability ranges for the solution (83)-(86) are determined from (28), (72) and (73). They are given by

$$
\begin{gathered}
0 \leq H<H_{0}, \quad N_{v}=0 \\
\sqrt{H_{N_{v}-1}^{2}-H_{S}^{2}} \leq H<H_{N_{v}}, \quad N_{v}=1,2, \ldots
\end{gathered}
$$

The upper bounds in (88), (89), $H_{N_{v}}\left(N_{v}=0,1,2, \ldots\right)$, are determined by the implicit equation

$$
\frac{L}{\lambda_{J}}=\left(N_{v}+1\right) \frac{H_{s}}{H_{N_{v}}} K\left(\frac{H_{s}^{2}}{H_{N_{v}}^{2}}\right), \quad N_{v}=0,1,2, \ldots,
$$

where $H_{s}=\left(e p \lambda_{J}\right)^{-1}$ is the superheating field of the Meissner state in a semi-infinite $(0 \leq y<\infty)$ layered superconductor [1,2], and $H_{0} \equiv H_{s L}>H_{s}$ is the superheating field of the Meissner state for $L<\infty$. Upon substitution of (83)-(86) into (78) (with $\bar{f} \equiv 1$, $\bar{\phi} \equiv \phi)$, one can verify the lower-bound estimates (74), (75). In Appendix B, we show that the solution (83)-(89) satisfies the Jacobi-Weierstrass - Hilbert sufficient condition for a strong minimum [19] of (51) [and hence of (3)].

Note that equation (82) was first analyzed in the context of a single Josephson junction a long time ago $[26,27]$. It was discussed in numerous subsequent publications [28]. Unfortunately, the complete, exact, closed-form solution (83)-(89), valid for arbitrary values of $L>0$ and $H \geq 0$, has not been obtained up until now. This situation manifested itself in the absence of any clear mathematical definition of the Josephson vortex at $H>0$ and gave rise to the erroneous belief $[15,16]$ that Josephson vortices «do not exist» for $L<<\lambda_{J}$.

Equations (83)-(89) provide an explicit form for the complete set of minimizers of the LD model (3) with $r(T)<<1$ and generalize the results of Refs. 1, 2 . For $N_{v}=1,2, \ldots$, they provide a complete set of soliton solutions to the coupled static SG equations (A1) and establish a one-to-one correspondence between vortex planes in layered superconductors and 
ordinary Josephson vortices in the single junction. (The only difference lies in the definition of the Josephson length $\lambda_{J}$.) For these reasons, the properties of (83)-(89) are of crucial importance.

Equations (83)-(86) reflect a general soliton feature: Solutions (83), (84) with even $N_{v}$ cannot be continuously transformed into solutions (85), (86) with odd $N_{v}$ by changing $H$ and vice versa. Solutions with $N_{v} \geq 1$ are pure solitons (vortex planes) only at $H=\sqrt{H_{N_{v}-1}^{2}-H_{s}^{2}}$, which corresponds to the boundary conditions (72). In the rest of the regions (88), we have solitons «dressed» by the Meissner field. (The Meissner and the vortex fields cannot be separated from each other, because the principle of superposition does not apply to the nonlinear equations (A1). Unfortunately, this important issue was not understood in previous publications [29].)

Of special interest is the overlap of the regions (87), (88) for $N_{v}=\bar{N}_{v}$ and $N_{v}=\bar{N}_{v}+1$. Owing to this property, the solutions obtained cover the whole field range $0 \leq H<\infty$, as they should. The overlap practically vanishes for $H_{N_{v}} \gg H_{s}$. Given that all $H_{N_{v}}$ decrease when $W=2 L$ increases, the overlap is stronger for large $W$ and can involve several neighboring states. As explained in the Introduction, the actual equilibrium state is the one that corresponds to the minimum of the thermodynamic Gibbs free energy for given $H$. A transition from the state with $N_{v}=\bar{N}_{v}$ to the state $N_{v}=\bar{N}_{v}+1$ with lower Gibbs free energy is a phase transition of the first-order type $[1,2]$. In particular, the lower critical field $H_{c 1}$ is determined from the requirement that the Gibbs free energy of the state $N_{v}=1$ be equal to that of the Meissner state $\left(N_{v}=0\right)$ and satisfy the relation $\sqrt{H_{s L}^{2}-H_{s}^{2}}<H_{c 1}<H_{s L}$. In the case $\lambda_{J}<<L<\infty$, it is given by $H_{c 1}=2 H_{s} / \pi[1,2]$.

Equations (83)-(89) contain corresponding results of Refs. 1, 2 as limiting cases. For example, by making the change of variable $y \rightarrow y-L$ and proceeding to the limit $L \rightarrow \infty$ in Eqs. (83), (84) with $N_{v}=0$, we obtain the exact Meissner solution in the semi-infinite interval $y \in[0,+\infty)$ :

$$
\phi(y)=-4 \arctan \frac{H \exp \left[-y / \lambda_{J}\right]}{H_{s}+\sqrt{H_{s}^{2}-H^{2}}} .
$$

By proceeding to the limit $L \rightarrow \infty$ in Eqs. (85), (86) with $N_{v}=1$, we arrive at the vortex-plane solution in the infinite interval $y \in(-\infty,+\infty)$ :

$$
\phi(y)=4 \arctan \exp \left[y / \lambda_{J}\right] .
$$

When the screening by Josephson currents is negligibly small, i.e., (i) for $L<<\lambda_{J}$ and arbitrary $H$, or (ii) for $H_{S}<<H$ and arbitrary $L$, equations (83)-(89) become

$$
\begin{gathered}
\phi(y)=\pi N_{v}+2 e p H y- \\
-\frac{(-1)^{N_{v}}}{4 e^{2} p^{2} \lambda_{J}^{2} H^{2}}[\sin (2 e p H y)-2 e p H y \cos (e p H W)],
\end{gathered}
$$

where $N_{v}=[e p H W / \pi]$ [see (69)]. The overlap of states with different $N_{v}$ now practically vanishes, and the period of the vortex structure for $N_{v} \geq 1$ is $P=\pi / e p H$, which refutes the claims $[15,16]$ that Josephson vortices «do not exist» in the limit $L<<\lambda_{J}$.

The substitution of (92) into (78) gives the equilibrium value of the LD free-energy functional (the thermodynamic free energy):

$$
\begin{gathered}
\Omega_{L D}(H)=\frac{H_{C}^{2}(T) p N L W_{z}}{4 \pi} \times \\
\times\left[-1+r(T)\left[1-\frac{|\sin (e p H W)|}{e p H W}+\frac{\cos ^{2}(e p H W)}{8\left(e p \lambda_{J} H\right)^{2}}\right]\right] .
\end{gathered}
$$

\section{Discussion}

We have obtained a complete, exact solution of the problem of the magnetic properties of layered superconductors with an infinite number of superconducting layers $(N=\infty)$ in parallel fields $H>0$, in the absence of transport currents. Based on a new exact variational method (Sec. 2.2, 2.3 and Appendix A), we have determined the type of all stationary points of the Gibbs free-energy functional (3) and the related Helmholtz free-energy functional [derived from (3) by setting $H=0]$ : For the Gibbs free-energy functional, they are either points of strict, strong minima or saddle points. All stationary points of the Helmholtz free-energy functional are those of strict, strong minima.

By evaluating the surface variation of the Helmholtz free-energy functional, we have found a complete set of stable, equilibrium field configurations: Namely, the Meissner (0-soliton or «vacuum») solution and soliton (vortex-plane) solutions. These solutions conserve the flux and realize exact lower bounds of the Helmholtz free-energy functional in the corresponding topological sectors. As shown in Appendix A, the absence of soliton solutions of the «fluxon» and «lattice» types at $H>0$ is due to the boundary conditions on the derivatives of the phase differences [Eqs. (27), (28)] that require the continuity of the local field at the outer interfaces. Physically, the fact that a vortex plane locally minimizes the free-energy functionals means that, contrary to a widespread be- 
lief [4], the effective interaction between flux quanta positioned in different insulating layers is attractive. Topological methods of Sec. 2.4 establish the true mathematical analogy between the vortex structure in continuum type-II superconductors and that in layered superconductors at $H>0$ : It consists in an isomorphism between a single Abrikosov vortex and a single vortex plane. (Note the role of the conservation law for the flux in the derivation of these results: Such conservation laws in nonlinear field theories always yield soliton solutions that minimize the energy functionals $[10-13]$.

In the limit of weak interlayer coupling, $r(T)<<1$, we have derived exact, closed-form analytical expressions for all stable solutions [Eqs. (83)-(89)]. Solutions (83)-(89) explicitly satisfy the SG equations (A1) with a full set of boundary conditions. They meet the Legendre necessary and Jacobi-Weierstrass - Hilbert sufficient conditions for a strong minimum [19] and contain the exact results of Refs. 1, 2 as particular limiting cases. Expressions (83)-(89) provide an adequate description of real physical systems with $\left.N>{ }^{-1}\right], \varepsilon<<1$ (see Appendix A), when boundary effects along the layering axis can be neglected.

We can now answer the question what kind of configurations correspond to saddle points of (3). As shown in Sec. 2.3 and Appendix A, all saddle-point configurations are non-soliton and violate the conservation law for the flux. Saddle points of the first type appear if one increases the external field $H$ beyond the stability regions (87), (88). (This type of saddle points exists already in the case of a single Josephson junction.) Saddle points of the second type appear as solutions to the boundary value problem (27), (28), (A18) for (A1), where the $Z_{n}$ violate (A22). Nonsoliton solutions of this type, interpreted as «vortex lattices», were considered in some previous publications on the LD model $[6,7,14]$ : As shown in Appendix $\mathrm{C}$, they are just perturbations of the soliton solutions (83)-(86) with $N_{v}>0$. Note that non-soliton numerical solutions $[16,17]$ for finite $(N<\infty)$ Josephson-junction stacks, interpreted as «isolated fluxons», belong to the same type: They are characterized by the condition

$$
\left[\frac{1}{2 \pi} \int_{-L}^{L} d y \frac{d \phi_{n}(y)}{d y}\right]=0
$$

for all $n$ and thus constitute perturbations of the Meissner solution (83), (84) with $N_{v}=0$.

It is instructive to compare our mathematical approach with previous approaches. Both in Refs. 1, 2 and in the present paper, we start by exact minimization of the Gibbs free-energy functional. By determining a complete set of the minimizers, we arrive at a natural physical interpretation of all relevant mathematical relations and the identification of equilibrium Josephson vortices as topological solitons (vortex planes). [In the weak-coupling limit, they are just the soliton solutions to the SG equations (A1).] In contrast, previous publications on the LD model started with an a priori assumption that the vortex structure in layered superconductors resembled that in continuum type-II superconductors [4-9]. Unfortunately, similarities were erroneously sought in spatial distribution of field configurations. However, unlike the true analogy in terms of homotopy theory (Sec. 2.4), any analogy in the configurational space is precluded by fundamental differences between the Gibbs free-energy functional (3) and that of continuum type-II superconductors.

We have already pointed out (Refs. 1, 2 and the Introduction) the inadequacy of mathematical methods of previous publications. It should be added that the necessity of ensuring the vanishing of the surface variation in the stationarity condition for the Helmholtz free-energy functional was not taken into account [4,7-9]: As a result, the conservation law for the flux and soliton solutions were lost. Since exact solvability and soliton solutions of the SG equations (A1) were not noticed, any mathematical definition of the $\mathrm{JO}^{-}$ sephson vortex could not be given. This situation has led to confusion as to what might be called the Josephson vortex at $H>0$ even in the simplest case of the single junction: Hence the erroneous claims $[15,16]$ that Josephson vortices «do not exist» for $L<<\lambda_{J}$.

The problem of the stability of the proposed nonsoliton configurations (i.e., whether they correspond to any points of minima of the Gibbs free-energy functional) has never been posed in previous theoretical publications. (This fact is not surprising in view of the neglect of the conservation law for the flux and accompanying uncontrolled mathematical approximations $^{\star}$.) Unfortunately, the issue of stability was disregarded in numerical simulations [16] for finite $(N<\infty)$ Josephson-junction stacks as well: Hence a misunderstanding [17] of the profound physical and

* For example, as shown by Farid [30], field configurations of the type of Refs. 4, 5 do not constitute any solutions in a true mathematical sense. We add that equations for the phase differences (A1) [let alone the more general equations (9), (10), (26)-(28)] were not derived in Refs. 4, 5. 
mathematical difference between soliton and nonsoliton solutions.

In summary, the central result of this paper is that equilibrium Josephson vortices in layered superconductors with $N=\infty$ are topological solitons of the static SG equations for the phase differences. This result should be viewed in the general context of vortex solutions in nonlinear field theories [10-13]. (For example, Abrikosov vortices in continuum type-II superconductors are topological solitons of the GL equations.) Mathematically, the exact, closed-form expressions (83)-(89) represent a new class of soliton solutions. Aside from their importance for weak superconductivity, they can find applications in different fields of nonlinear physics and applied mathematics where the SG equations are involved [11].

\section{Appendix A}

\section{The solution of coupled static SG equations}

In the limit of weak coupling, considered in Sec. 3, the zeroth-order [with respect to $r(T)<<1$ ] solution to (9), (10) has the form $f_{n} \equiv 1$. Upon substitution into Eqs. (26) and subsequent differentiation with respect to $y$, the latter equations are reduced to coupled static SG equations

$$
\lambda_{J}^{2} \frac{d^{2} \phi_{n}(y)}{d y^{2}}=\frac{1}{\varepsilon^{2}} \sum_{m} G^{-1}(n, m) \sin \phi_{m}(y), n=1, \ldots, N,
$$

where $G^{-1}(n, m)$ is a Jacobian matrix [31] with elements $\quad G^{-1}(n, n)=2+\varepsilon^{2} \quad(n=1, \ldots, N)$, $G^{-1}(n+1, n)=G^{-1}(n, n+1)=-1 \quad(n=1, \ldots, N-1)$, and $G^{-1}(n, m)=0$ for $|n-m|>1$. Owing to periodic boundary conditions

$$
\phi_{n+N}(y)=\phi_{n}(y)
$$

[see (6)], the matrix $G^{-1}(n, m)$ is cyclic. Equations (A1) are subject to conditions (27), (28), and their solutions obey the symmetry relations (25).

In the limit $N \rightarrow \infty$, equations (A1) were derived by a different method in a number of publications $[6,7]$. Unfortunately, their analytical properties have not been studied. The main property can be formulated as the following proposition:

Proposition. Consider Eqs. (A1) on the whole axis $-\infty<y<+\infty$. The initial value problem for Eqs. (A1) with arbitrary initial conditions

$$
\phi_{n}\left(y_{0}\right)=\alpha_{n}, \quad \frac{d \phi_{n}}{d y}\left(y_{0}\right)=\beta_{n} \quad\left(\left|y_{0}\right|<\infty\right)
$$

has a unique solution in the whole interval $-\infty<y<+\infty$. This solution has continuous deriva- tives with respect to $y$ of arbitrary order and depends continuously on the initial data.

To prove the Proposition, we note that Eqs. (A1) satisfy the conditions of Picard's theorem on the existence and uniqueness of a global solution [32]. This property is rather unusual for nonlinear differential equations: Global character of the solution and its infinite differentiability are ensured by the fact that the $\phi_{n}$ enter the right-hand side of Eqs. (A1) only as arguments of the sine.

Equations (A1) can be rewritten in an equivalent form:

$$
\sin \phi_{n}(y)=\varepsilon^{2} \lambda_{J}^{2} \sum_{m} G(n, m) \frac{d^{2} \phi_{m}(y)}{d y^{2}}, n=1, \ldots, N .
$$

The matrix $G(n, m)$, being the inverse of $G^{-1}(n, m)$, has the form

$$
G(n, m)=\frac{\mu^{|n-m|}}{2 \varepsilon \sqrt{1+\varepsilon^{2} / 4}}, \quad \mu=1+\frac{\varepsilon^{2}}{2}-\varepsilon \sqrt{1+\varepsilon^{2} / 4},
$$

and obeys the summation rule

$$
\sum_{m} G(n, m)=\frac{1}{\varepsilon^{2}}
$$

The matrix $G(n, m)$ is positive definite, because all its eigenvalues $e_{k}$ are positive:

$$
\begin{gathered}
e_{k}=\frac{\lambda_{k}^{2}}{\varepsilon^{2} \lambda_{J}^{2}}, \quad \lambda_{k}=\frac{\varepsilon \lambda_{J}}{\sqrt{2+\varepsilon^{2}-2 \cos (2 \pi k / N)}}, \\
k=0, \pm 1, \ldots,\left[\frac{N}{2}\right] .
\end{gathered}
$$

The quantities $\lambda_{k}$ in (A6) are the characteristic length scales of Eqs. (A1). [Note that (A1) are characterized by a distribution of length scales, not just two length scales, as is claimed in some previous publications.] The distribution of the length scales becomes quasicontinuous under the condition

$$
N>>\left[\varepsilon^{-1}\right],
$$

which can be regarded as a criterion of the applicability of the LD model to layered superconductors.

The fact that equilibrium solutions (83)-(89) correspond to the largest length scale $\lambda_{\max } \equiv \lambda_{0} \equiv \lambda_{J}$ is by no means surprising: In equilibrium, the system tends to minimize a diamagnetic response to the external field $H$. Note that for $N \rightarrow \infty, H=0, L=\infty$, equations (A1) admit an exact soliton - antisoliton solution

$$
\phi_{n}(y)=(-1)^{n} \phi(y), \quad \phi(y)=4 \arctan \exp \left(\frac{y}{\lambda_{\min }}\right),
$$




$$
\lambda_{\min } \equiv \lambda_{[N / 2]}=\frac{\varepsilon \lambda_{J}}{\sqrt{4+\varepsilon^{2}}} .
$$

However, solution (A8), (A9) vanishes for any $H>0$, $L<\infty$.

Note the first integral of (A1):

$\sum_{n} \cos \phi_{n}(y)+\frac{\varepsilon^{2} \lambda_{J}^{2}}{2} \sum_{n} \sum_{m} G(n, m) \frac{d \phi_{n}(y)}{d y} \frac{d \phi_{m}(y)}{d y}=C$.

(A10)

In the case of a finite interval $y \in[-L, L]$, the constant of integration $C$ can be determined from the conditions (27), (28):

$$
C=\frac{2 N H^{2}}{H_{s}^{2}}+\sum_{n} \cos \phi_{n}(-L) .
$$

In the infinite interval $y \in(-\infty,+\infty), C=N$.

Now, we will prove that Eqs. (83)-(89) provide a complete set of stable solutions to (A1) at $H>0$. Using equations of Secs. 2.1, 2.2 with $f_{n} \equiv 1$ and introducing the «local magnetic field» in the regions $(n-1) p \leq x<n p$ via

$$
h_{n}(y)=\frac{\varepsilon^{2}}{2 e p} \sum_{m} G(n, m) \frac{d \phi_{m}(y)}{d y},
$$

we rewrite (3) as follows:

$$
\begin{gathered}
\Omega_{L D}\left[\phi_{n}, \frac{d \phi_{n}}{d y} ; H\right]=\Omega_{0}+\Omega^{*}\left[\phi_{n}, \frac{d \phi_{n}}{d y} ; H\right], \quad \text { (A13) } \\
\Omega^{*}\left[\phi_{n}, \frac{d \phi_{n}}{d y} ; H\right]=\frac{p W_{z}}{8 \pi} \sum_{n} \int_{-L}^{L} d y\left[\frac{H_{s}^{2}}{2}\left[1-\cos \phi_{n}(y)\right]+\right. \\
\left.+\frac{1}{\varepsilon^{2}}\left[h_{n+1}(y)-h_{n}(y)\right]^{2}+\left[h_{n}(y)-H\right]^{2}\right]= \\
=\frac{N p W W_{z}^{2} H}{8 \pi}+\Omega_{H}^{*}\left[\phi_{n}, \frac{d \phi_{n}}{d y}\right]-\frac{H \Phi W_{z}}{4 \pi} \geq 0, \text { (A14) }
\end{gathered}
$$

where

$$
\begin{gathered}
\Omega_{H}^{*}\left[\phi_{n}, \frac{d \phi_{n}}{d y}\right]=\frac{H_{s}^{2} p W_{z}}{16 \pi} \int_{-L}^{L} d y\left[\sum_{n}\left[1-\cos \phi_{n}(y)\right]+\right. \\
\left.+\frac{\varepsilon^{2} \lambda_{J}^{2}}{2} \sum_{n} \sum_{m} G(n, m) \frac{d \phi_{n}(y)}{d y} \frac{d \phi_{m}(y)}{d y}\right] \geq 0 \quad \text { (A15 }
\end{gathered}
$$

is the Helmholtz free-energy functional, and the total flux is given by

$$
\Phi=\frac{1}{2 e} \sum_{n}\left[\phi_{n}(L)-\phi_{n}(-L)\right]
$$

The treatment of the functionals $\Omega^{*}, \Omega_{H}^{*}$ is fully analogous to that of $\Omega, \Omega_{H}$ in Sec. 2.3. Thus, by virtue of positive definitiveness of (A4), the Legendre necessary condition for a strong minimum [19],

$$
\sum_{n} \sum_{m} G(n, m) \rho_{n} \rho_{m}>0, \quad \sum_{n} \rho_{n}^{2} \neq 0
$$

where $\rho_{n}$ are arbitrary real numbers, is explicitly fulfilled. The functionals $\Omega^{*}, \Omega_{H}^{*}$ have a common set of minimizers. The stationarity condition for $\Omega_{H}^{*}$ involves the vanishing of the volume variation, which yields Eqs. (A3), and of the surface variation, which leads, by (27) and (A16), to the conservation law for the flux $\delta \Phi=0$. By analogy with (69), (70), we arrive at soliton boundary conditions

$$
\begin{array}{r}
\frac{\phi_{n}(0)}{\pi}=Z_{n} \equiv\left[\frac{1}{2 \pi} \int_{-L}^{L} d y \frac{d \phi_{n}(y)}{d y}\right] \geq 0(n=1, \ldots, N), \\
-\frac{\phi_{n}(-L)}{\pi} \equiv\left\{\frac{1}{2 \pi} \int_{-L}^{L} d y \frac{d \phi_{n}(y)}{d y}\right\}, \quad-\pi<\phi_{n}(-L) \leq 0 .
\end{array}
$$

The soliton solution in the sector $\left\{Z_{1}, \ldots, Z_{N}\right\}$ first appears under $N$ conditions

$$
\phi_{1}(-L)=\ldots=\phi_{N}(-L)=0
$$

[see (A19)] and $N-1$ conditions

$$
\frac{d \phi_{1}}{d y}(-L)=\ldots=\frac{d \phi_{N}}{d y}(-L)=2 e p H^{*} \geq 0
$$

where the field $H^{*}$ is so far undetermined [compare with (27), (28)]. Given that the general solution to (A1) contains $2 \mathrm{~N}$ constants of integration, conditions (A20), (A21) leave only one undetermined constant to satisfy the boundary conditions (A18). For this reason, we have to set

$$
Z_{1}=\ldots Z_{N}=Z \equiv N_{v}=0,1, \ldots
$$

For $H^{*}=0$, by the Proposition, the unique solution to the initial value problem (A20), (A21) in the interval $y \in[-L, L]$ is the trivial Meissner configuration $\phi_{1}=\ldots=\phi_{N} \equiv 0, N_{v}=0$. For arbitrary $H^{*}>0$, by the Proposition, the initial value problem (A20), (A21) also admits a unique solution in the interval $y \in[-L, L]$, and its explicit form is

$$
\begin{gathered}
\phi_{1}(y)=\ldots=\phi_{N}(y) \equiv \phi(y)= \\
=-\pi+2 \mathrm{am}\left(\frac{y+L}{k \lambda_{J}}+K\left(k^{2}\right), k\right),
\end{gathered}
$$




$$
k=\frac{H_{s}}{\sqrt{H^{* 2}+H_{S}^{2}}} .
$$

Upon substitution of (A23), (27) into (A18), with (A22) and $N_{v}=1,2, \ldots$, we determine $H^{*}=$ $=\sqrt{H_{N_{v}-1}^{2}-H_{s}^{2}}$, where $H_{N_{v}}$ are given by (41). In this way, we arrive at the solutions (83)-(89), which proves their uniqueness as minimizers of (A13)(A15). This proof clearly demonstrates that the absence of soliton solutions of any other types is a result of the physical boundary conditions (A21) [or (27), (28)].

To establish a relationship to the exact variational method of Refs. 1, 2, we note that (83)-(89) can also be obtained by the minimization of (A13)-(A15) with respect to the phases $\varphi_{n}$. (We recall that $\phi_{n} \equiv \varphi_{n}-$ $-\varphi_{n-1}$.) However, as first noticed in Refs. 1, 2, we must take into account that not all $\varphi_{n}$ are independent: The conservation law for the current (22) constitutes a constraint on $d \varphi_{n} / d y$. This problem can be easily circumvented by making use of the first integral (A10), (A11). The substitution of (A10), (A11) into (A15) yields

$$
\begin{gathered}
\Omega_{H}^{*}\left[\phi_{n} ; \phi_{n}(-L), \frac{d \phi}{d y}(-L)\right]=\frac{N W W_{z}}{32 \pi e^{2} p}\left[\frac{d \phi}{d y}(-L)\right]^{2}+ \\
+\frac{H_{s}^{2} p W_{z}}{16 \pi} \int_{-L}^{L} d y \sum_{n}\left[1-2 \cos \phi_{n}(y)+\cos \phi_{n}(-L)\right] .
\end{gathered}
$$

Taking the variation of (A25) with respect to $\varphi_{n}$, we immediately arrive at the relations

$$
\sin \phi_{n}(y)=\sin \phi_{n+1}(y)
$$

[compare with (40)], hence the condition (A22) and Eqs. (83)-(89).

\section{Appendix B}

\section{A verification of the Jacobi-Weierstrass-Hilbert sufficient condition for a strong minimum}

In the weak-coupling $\operatorname{limit} r(T)<<1$, when $f(y) \equiv 1$, the Legendre necessary condition of the strong minimum for $\Omega_{H}$ reduces to the second relation (54). Complemented by the requirement that the explicit solution (83)-(89) can be embedded into a field of extremals, this condition becomes the Jacobi-Weierstrass-Hilbert sufficient condition for a strong minimum [19].

In view of the symmetry relation $\phi(y)=-\phi(-y)+$ $+2 \pi N_{v}$ and the conditions on variations $\delta \phi( \pm L)=0$, $\delta \phi(0)=0$, it suffices to verify the Jacobi-Weierstrass - Hilbert condition for $y \in[-L, 0]$. At $H>0$, the desired field is given by the one-parameter family

$$
\begin{gathered}
\phi(y, \beta)=\pi\left(N_{v}-1\right)+ \\
+2 \mathrm{am}\left(\frac{y \sqrt{4+\beta^{2}}}{2 \lambda_{J}}+K\left(\frac{4}{4+\beta^{2}}\right), \frac{2}{\sqrt{4+\beta^{2}}}\right), \\
N_{v}=2 m, m=0,1, \ldots ; \\
\phi(y, \beta)=\pi N_{v}+2 \mathrm{am}\left(\frac{y \beta}{2 \lambda_{J}}, 2 \beta\right), \\
N_{v}=2 m+1, m=0,1, \ldots,
\end{gathered}
$$

where $\beta>0$. The family $\phi(y, \beta)$ explicitly satisfies (82) and the initial conditions

$$
\phi(0, \beta)=\pi N_{v} \frac{d \phi}{d y}(0, \beta)=\frac{\beta}{\lambda_{J}} .
$$

For $\beta=\bar{\beta}$, where $\bar{\beta}=2 \sqrt{1-k^{2}} / k$ in the case (B1), and $\bar{\beta}=2 / k$ in the case (B2), we have $\phi(y, \bar{\beta})=\phi(y)$, i.e., relations (B1) and (B2) yield the solutions (83), (84) and (85), (86), respectively. To prove that the family $\phi(y, \beta)$ indeed forms a field of extremals, we have to show that any two representatives $\phi_{1} \equiv \phi\left(y, \beta_{1}\right)$ and $\phi_{2} \equiv \phi\left(y, \beta_{2}\right)$, where $0<\beta_{1}<\beta_{2}$, do not intersect in the interval $[-L, 0)$. From the first integral of (82) [equation (76) with $f \equiv 1$ ] and (B3), we have

$$
\begin{aligned}
& \frac{\lambda_{J}^{2}}{2}\left(\frac{d \phi_{1}}{d y}\right)^{2}+\cos \phi_{1}=\frac{\beta_{1}^{2}}{2}+(-1)^{N_{v}}, \\
& \frac{\lambda_{J}^{2}}{2}\left(\frac{d \phi_{2}}{d y}\right)^{2}+\cos \phi_{2}=\frac{\beta_{2}^{2}}{2}+(-1)^{N_{v}} .
\end{aligned}
$$

We will prove the absence of points of intersection of $\phi_{1}$ and $\phi_{2}$ by contradiction.

Consider $\psi(y) \equiv \phi_{2}(y)-\phi_{1}(y)$. As Eq. (82) yields

$$
\frac{d^{2} \phi_{1}}{d y^{2}}(0)=\frac{d^{2} \phi_{2}}{d y^{2}}(0)=0,
$$

for $y \in\left(-\delta_{1}, 0\right)$, where $\delta_{1}$ is sufficiently small, we have

$$
\psi(y)=\left(\beta_{1}-\beta_{2}\right)|y|+o\left(y^{2}\right)>0 .
$$

By continuity of $\psi(y)$, relation (B5) implies the existence of a finite interval $y \in\left(y_{0}, 0\right)$ where $\psi(y)>0$. Let $y=y_{0} \in[-L, 0)$ be a point of intersection, i.e.,

$$
\phi_{1}\left(y_{0}\right)=\phi_{2}\left(y_{0}\right), \quad \frac{d^{2} \phi_{1}}{d y^{2}}\left(y_{0}\right)=\frac{d^{2} \phi_{2}}{d y^{2}}\left(y_{0}\right) .
$$


For $y \in\left(y_{0}, y_{0}+\delta\right)$, where $\delta>0$ is sufficiently small, we have

$\psi(y)=\left[\frac{d \phi_{2}}{d y}\left(y_{0}\right)-\frac{d \phi_{1}}{d y}\left(y_{0}\right)\right]\left(\left|y_{0}\right|-|y|\right)+o\left[\left(y-y_{0}\right)^{2}\right]>0$

which, in view of the arbitrariness of $\delta$, implies

$$
\frac{d \phi_{2}}{d y}\left(y_{0}\right) \geq \frac{d \phi_{1}}{d y}\left(y_{0}\right)
$$
However, equations (B4), by virtue of $\frac{d \phi_{1}}{d y}, \frac{d \phi_{2}}{d y}>0$,
yield

$$
\frac{d \phi_{2}}{d y}\left(y_{0}\right)<\frac{d \phi_{1}}{d y}\left(y_{0}\right) .
$$

The contradiction between (B6) and (B7) proves the absence of points of intersection in the whole interval $[-L, 0)$, as expected. Thus, the solution (83) $-(89)$, for any $H>0$, can be embedded into a field of extremals and, as such, satisfies the Jacobi-Weierstrass - Hilbert sufficient condition for a strong minimum.

\section{Appendix C}

A comparison between soliton and non-soliton («lattice») configurations

At fields

$$
H>\varepsilon^{-1} H_{s} \equiv\left(\varepsilon e p \lambda_{J}\right)^{-1},
$$

the SG equations (A1) with $N=2 m \rightarrow \infty$ admit an exact, closed-form analytical non-soliton solution under the conditions

$$
\frac{d \phi_{n}}{d y}( \pm L)=2 e p H, \quad \phi_{n}(0)=\pi n .
$$

A solution of this type was proposed, e.g., in Refs. 7, 14, where it was erroneously interpreted as a «dense triangular lattice of Josephson vortices». As an illustration of the general results of the paper, it is instructive to compare this solution with the exact closed-form analytical soliton solution (92), valid in a wider field range $H \gg H_{s}$.

By introducing a dimensionless variable $u \equiv 2 e p H y$ and new functions $\psi_{n}(u) \equiv \phi_{n}(u / 2 e p H)$, we rewrite Eqs. (A1) as

$$
\begin{gathered}
\frac{d^{2} \psi_{n}(u)}{d u^{2}}=\frac{1}{\left(2 \varepsilon e p \lambda_{J} H\right)^{2}} \sum_{m} G^{-1}(n, m) \sin \psi_{m}(u), \\
n=1, \ldots, N .
\end{gathered}
$$

The boundary conditions, Eqs. (C2), become

$$
\frac{d \psi_{n}}{d u}( \pm 2 e p H L)=1, \quad \phi_{n}(0)=\pi n .
$$

Taking into account (C1), we seek the solution to (C3), (C4) as an asymptotic expansion in powers of $1 /\left(2 e p \lambda_{J} H\right)^{2}$ :

$$
\psi_{n}(u)=\sum_{k \geq 0} \psi_{n}^{(k)}(u)
$$

where $\psi_{n}^{(k)}(u)$ is of order $1 /\left(2 \varepsilon e p \lambda_{J} H\right)^{2 k}$ $(k=0,1, \ldots)$. Retaining only the first two terms in (C5), we obtain

$$
\begin{aligned}
\phi_{n}(y)=\pi n & +2 e p H y-\frac{4+\varepsilon^{2}}{\varepsilon^{2}} \frac{(-1)^{n}}{4\left(e p \lambda_{J} H\right)^{2}} \sin (2 e p H y)+ \\
& +\frac{4+\varepsilon^{2}}{\varepsilon^{2}} \frac{(-1)^{n}}{2 e p \lambda_{J}^{2} H} \cos (e p H W) y . \quad \text { (C6) }
\end{aligned}
$$

The sum of the first three terms on the right hand side of (C6) in the limit $\varepsilon<<1$ gives the solution of Refs. 7, 14. The presence of the last term on the right-hand side of (C6), resulting from the boundary conditions (C2) at $y= \pm L$, was not noticed in Refs. 7 , 14, and therefore the solution of Refs. 7, 14 does not meet the boundary conditions at $y= \pm L$ in required order. In contrast to the exact closed-form analytical soliton solution (92), valid in the same field range and minimizing the LD functional (3), the non-soliton solution (C6) is just a saddle point of (3): see Secs. 2.3 and 4 and Appendix A. This is illustrated below.

The substitution of (C6) into (A13), (A14) yields a nonequilibrium value of the $\mathrm{LD}$ functional:

$$
\begin{gathered}
\tilde{\Omega}_{L D}(H)=\frac{H_{c}^{2}(T) p N L W_{z}}{4 \pi} \times \\
\times\left[-1+r(T)\left[1+\frac{\left[1+2 / \varepsilon^{2}\right] \cos ^{2}(e p H W)}{8\left(e p \lambda_{J} H\right)^{2}}\right]\right] .
\end{gathered}
$$

Expression (C7) is to be compared with the thermodynamic free energy (93) of the soliton solution (92). Their difference is

$$
\begin{gathered}
\Delta \tilde{\Omega}_{L D}(H) \equiv \tilde{\Omega}_{L D}(H)-\Omega_{L D}(H)= \\
=\frac{H_{C}^{2}(T) p N L W_{z} r(T)}{4 \pi} \times \\
\times\left[\frac{|\sin (e p H W)|}{e p H W}+\frac{\cos ^{2}(e p H W)}{4\left(e p \lambda_{J} H\right)^{2} \varepsilon^{2}}\right]>0 .
\end{gathered}
$$

Thus, the saddle-point, non-soliton solution (C6) is nothing but an unstable perturbation of the soliton solution (92). 
1. S.V. Kuplevakhsky, Phys. Rev. B60, 7496 (1999).

2. S.V. Kuplevakhsky, Phys. Rev. B63, 054508 (2001).

3. W.E. Lawrence and S. Doniach, in: Proceedings of the Twelfth Conference on Low Temperature Physics, Kyoto, 1970, E. Kanda (ed.), Keigaku, Tokyo (1970), p. 361 .

4. L.N. Bulaevskii, Zh. Eksp. Teor. Fiz. 64, 2241 (1973) [Sov. Phys. JETP 37, 1133 (1973)].

5. J.R. Clem and M.W. Coffey, Phys. Rev. B42, 6209 (1990).

6. J.P. Carton, J. Phys. I (France) 1, 113 (1991).

7. L.N. Bulaevskii and J.R. Clem, Phys. Rev. B44, 10234 (1991).

8. L.N. Bulaevskii, M. Ledvij, and V.G. Kogan, Phys. Rev. B46, 366 (1992).

9. A.E. Koshelev, Phys. Rev. B48, 1180 (1993).

10. R. Rajaraman, Solitons and Instantons, North-Holland, Amsterdam (1982).

11. R.K. Dodd, J.C. Eilbeck, J.D. Gibbon, and H.C. Morris, Solitons and Nonlinear Wave Equations, Academic Press, London (1982).

12. P. Goddard and P. Mansfield, Rep. Prog. Phys. 49, 725 (1986).

13. A.S. Schwarz, Quantum Field Theory and Topology Nauka, Moscow (1989) (in Russian).

14. L.N. Bulaevskii, M. Maley, H. Safar, and D. Dominguez, Phys. Rev. B53, 6634 (1996).

15. L.N. Bulaevskii, J.R. Clem, and L.I. Glazman, Phys. Rev. B46, 350 (1992)

16. V.M. Krasnov, Phys. Rev. B63, 064519 (2001).

17. V.M. Krasnov, Phys. Rev. B65, 096503 (2002).
18. C. Lancsos, The Variational Principles of Mechanics, University of Toronto Press, Toronto (1962).

19. N.I. Akhiezer, The Calculus of Variations, Blaisdell Publishing, New York (1962).

20. N.W. Ashcroft and N.D. Mermin, Solid State Physics, Holt, Rinehart and Winston, New York (1976).

21. A.O. Gelfond, The Calculus of Finite Difference, GIFML, Moscow (1959) (in Russian).

22. A.A. Abrikosov, Fundamentals of the Theory of $\mathrm{Me}$ tals, North-Holland, Amsterdam (1988).

23. V.E. Zakharov and E.A. Kuznetsov, Zh. Eksp. Teor. Fiz. 66, 594 (1974).

24. N.D. Mermin, Rev. Mod. Phys. 51, 591 (1979).

25. M. Abramowitz and I.A. Stegun, Handbook of Mathematical Functions, Dover, New York (1965).

26. I.O. Kulik, Zh. Eksp. Teor. Fiz. 51, 1953 (1966) [Sov. Phys. JETP 24, 1307 (1967)].

27. C.S. Owen and D.J. Scalapino, Phys. Rev. 164, 538 (1967).

28. A. Barone and G. Paterno, Physics and Applications of the Josephson Effect, Wiley, New York (1982).

29. A. Buzdin and D. Feinberg, Phys. Lett. A165, 281 (1992).

30. B. Farid, J. Phys. Condens. Matter 10, L589 (1998)].

31. R. Bellman, Introduction to Matrix Analysis, McGrawHill, New York (1960).

32. V.C.L. Hutson and J.S. Pym, Applications of Functional Analysis and Operator Theory, Academic Press, London (1980). 